\title{
Characterizing the transmission patterns of seasonal influenza in Italy: lessons from the last decade
}

\author{
Filippo Trentini ${ }^{1,2^{*}}$, Elena Pariani ${ }^{3}$, Antonino Bella ${ }^{4}$, Giulio Diurno ${ }^{5}$, Lucia Crottogini ${ }^{6}$, Caterina Rizzo ${ }^{7 \dagger}$, \\ Stefano Merler ${ }^{1+}$ and Marco Ajelli ${ }^{8+}$
}

\begin{abstract}
Background: Despite thousands of influenza cases annually recorded by surveillance systems around the globe, estimating the transmission patterns of seasonal influenza is challenging.

Methods: We develop an age-structured mathematical model to influenza transmission to analyze ten consecutive seasons (from 2010 to 2011 to 2019-2020) of influenza epidemiological and virological data reported to the Italian surveillance system.

Results: We estimate that $18.4-29.3 \%$ of influenza infections are detected by the surveillance system. Influenza infection attack rate varied between 12.7 and $30.5 \%$ and is generally larger for seasons characterized by the circulation of A/H3N2 and/or B types/subtypes. Individuals aged 14years or less are the most affected age-segment of the population, with $A$ viruses especially affecting children aged $0-4$ years. For all influenza types/subtypes, the mean effective reproduction number is estimated to be generally in the range 1.09-1.33 (9 out of 10 seasons) and never exceeding 1.41. The age-specific susceptibility to infection appears to be a type/subtype-specific feature.

Conclusions: The results presented in this study provide insights on type/subtype-specific transmission patterns of seasonal influenza that could be instrumental to fine-tune immunization strategies and non-pharmaceutical interventions aimed at limiting seasonal influenza spread and burden.
\end{abstract}

Keywords: Seasonal influenza, Burden, Susceptibility to infection, Eproduction number

\section{Introduction}

Annual influenza epidemics cause a marked excess of mortality and hospitalization as well as significant economic and healthcare burden [1]. Worldwide, influenzaassociated respiratory deaths are estimated to be in the range $4.0-8.8$ per 100,000 individuals, with a heavier burden of 17.9-223.5 deaths per 100,000 people among individuals aged 75 years or more [2]. To monitor influenza

\footnotetext{
*Correspondence: ftrentini@fbk.eu

${ }^{\dagger}$ Caterina Rizzo, Stefano Merler and Marco Ajelli joint senior authors.

${ }^{1}$ Center for Health Emergencies, Bruno Kessler Foundation, Trento, Italy

Full list of author information is available at the end of the article
}

spread, surveillance is carried out worldwide by means of records of patients showing influenza-like illness (ILI) and virological investigation of circulating types/subtypes. Moreover, due to the continuous antigenic changes of the virus, the investigation of the circulating influenza types and subtypes and the assessment of their burden in specific age classes across different seasons is crucial to update the composition of the vaccine and increase its efficacy [3]. However, due to the low proportion of individuals developing clinical symptoms after influenza infection [4] and thus the low proportion of individuals consulting general practitioners [5], the picture returned 
by the surveillance data is far from being representative of the true influenza burden.

Mathematical models of infectious disease transmission represent key tools to properly interpret the observed data and to provide quantitative estimates of quantities that hard to measure directly [6-9]. In this study, we use mathematical modeling to estimate three key epidemiological indicators: i) the influenza infection attack rate (overall and by age), which corresponds to the proportion of individuals infected by influenza over the entire course of the season; ii) the effective reproductive number, $R_{\text {eff }}$ - i.e., the mean number of secondary infections caused by a typical infectious individual in a partially immune population; and iii) the age-specific susceptibility to infection by virus type/subtype. Each epidemiological indicator is estimated for ten influenza seasons (from 2010 to 2011 to 2019-2020) in Italy and for each circulating influenza type/subtype.

Modeling and comparing the influenza types/subtypes allows us elucidating type/subtype-specific features, such as the age-specific susceptibility to infection, and to what extent the co-circulation of different types/subtypes alters the transmission patterns at the population level of the single type/subtype, measured in terms of the effective reproduction number. The estimates provided in this study shed new light on the transmission dynamics of seasonal influenza, showing that the total infection attack rate has a low variability across different seasons, the reproduction number is not markedly different by influenza type/subtype, and underage individuals play a central role in spreading the infection.

\section{Methods}

\section{Influenza like illness and virological surveillance data}

We analyze the data reported to the Italian epidemiological and virological influenza surveillance system from 2010 to 2011 to 2019-2020. Briefly, sentinel GPs (general practitioners (GPs) and pediatricians (PDs)) are asked to report weekly influenza like illness cases (ILI, defined as acute onset of fever $>38^{\circ} \mathrm{C},+$ respiratory symptoms + one of these symptoms: headache, general discomfort, asthenia) occurring during the year, from week 42 to week 17, using standardized forms. Specific information regarding age (0-4, 5-14, 15-64, > 64 years) and influenza vaccine status are collected and reported using web-based electronic Case Report Forms [10]. GPs and PDs are also able to define their assisted population by age group because every individual in Italy has to be appointed to a specific GP through the regional health service. This measure provides the denominator to calculate the incidence of ILI cases by age group at the National and regional level (every year $2 \%$ of the regional population is requested to be under surveillance as per indication of the National Public Health Institute that coordinates the surveillance scheme).

\section{Virological surveillance data}

For surveillance of circulating influenza viruses - sampling kits are sent out to regional coordinator for surveillance that randomly select sentinel GPs during the weeks between week 46 and week 17 to collect throat swabs among ILI-patients. The number of randomly selected swabs is proportional to the weekly incidence of reported ILIs [see Fig.S1 in Additional File 1]. These swabs are analyzed at the regional Reference Laboratories distributed in 15 different Italian regions [11]. Results are collected and reported using web-based electronic CFR from the National Influenza Centre (NIC). Every season, a subset of samples of ILI-patients are collected and analyzed (e.g. antigenic and genetic characterization) by the Italian National Institute of Health (ISS), with a proportion of positive specimens between 20 and 40\% [12].

Virological data from the Italian region of Lombardy for the 2010-2011 season [13] and from the National Influenza Surveillance Scheme from 2011 to 2012 to 2019-2020 season [14] are used in our analysis. A comparison between national virological surveillance and virological data for Italian region of Lombardy from 2010 to 2011 to 2016-2017 season is reported in the Appendix [see Fig.S2 in Additional File 1].

\section{Seroepidemiological data}

We analyze seroepidemiological data collected before and at the end of the $2009 \mathrm{~A} / \mathrm{H} 1 \mathrm{~N} 1$ pandemic to assess both the pre- [15] and post- [16] pandemic susceptibility to infection and the level of immunity by age-group to the $2009 \mathrm{~A} / \mathrm{H} 1 \mathrm{~N} 1$ influenza virus in the Italian general population. The level of immunity against the 2009 $\mathrm{A} / \mathrm{H} 1 \mathrm{~N} 1$ influenza virus in pre- and post-pandemic sera are determined using left over sera taken for diagnostic purposes or routine ascertainment obtained from clinical laboratories. The antibody titres are measured by the haemagglutination inhibition (HI) assay (the presence of protective antibody $(\geq 1: 40)$, are calculated using exact binomial $95 \%$ CI on both pre- and post- pandemic serological data) [16].

For each season we consider two datasets: i) the weekly incidence of ILI cases by age group (four age groups: 0-4 years, 5-14years, 15-64 years, and 65+ years) [17], and ii) the share of samples collected among ILI cases testing positive for each of three influenza types/subtypes (namely, A/H1N1pdm09, A/H3N2, and B - both Victoria and Yamagata) by age (same four age groups of the ILI cases) [13, 14] and the seroepidemiological profile of the Italian population against $2009 \mathrm{H} 1 \mathrm{~N} 1$ influenza pandemic. 
In addition to the epidemiological data, for each season, we collect data about the influenza vaccination coverage by age group [18] and age structure of the Italian population [19]. Estimates of vaccine effectiveness by type/subtype are taken from Belongia et al. [20]. Details on the data used for the analyses are presented in the Appendix [see Tables S1-S4 in Additional File 1].

\section{Estimation of ILI reporting rate}

We subtract the proportion of samples positive for 2009 A/H1N1 pandemic virus (A/California/07/2009) identified after and before the $2009 \mathrm{~A} / \mathrm{H} 1 \mathrm{~N} 1$ pandemic virus circulated in Italy to obtain an estimate of the infection attack rate (AR) of the pandemic for individuals aged $0-14$ years and $15+$ years. We label these quantities as $A R_{0-14}$ and $A R_{15+}$, respectively. We then sum the weekly ILI cases reported to the surveillance system during the pandemic in the same two age groups and multiply it by the share of samples collected among ILI cases testing positive for $\mathrm{A} / \mathrm{H} 1 \mathrm{~N} 1 \mathrm{pdm} 09$. These quantities, denoted as $A R_{0-14}^{I L I}$ and $A R_{15+}^{I L I}$, essentially represent the attack rate detected by the surveillance system of the Italian National Institute of Health (ISS). Therefore, we can estimate the reporting rate of the surveillance system for the age group $0-14$ years and $15+$ years as the ratios $r_{0-14}=A R_{0-14}^{I L I} / A R_{0-14}$ and $r_{15+}=A R_{15+}^{I L I} / A R_{15+}$, respectively. Details are provided in the Appendix [see Section 3 in Additional File 1].

\section{Estimation of age-specific infection attack rates for seasonal influenza}

We leverage the estimated reporting rates by age of the surveillance system to estimate the infection attack rate of each type/subtype for each of the analyzed influenza seasons. In particular, we define $\operatorname{ILI}_{a}(w ; y)$ the incidence of ILI cases reported to the surveillance system for age group $a$ on week $w$ of season $y$. We also define $f_{a}(y, s)$ the share of ILI samples for age group $a$ testing positive for type/subtype $s$ in season $y$. Therefore, following the procedure presented in [16], we can estimate the incidence of influenza cases linked to type/ subtype $s$ for each age group and influenza season as $I L I_{a}^{s}(w ; y)=I L I_{a}(w ; y) * f_{a}(y, s)$. By summing over all weeks, we can get an estimate to the age-specific AR detected by the surveillance system. Using the reporting rate by age of the surveillance system (see previous section), we can estimate the influenza infection AR by age in season $y$ as $A R_{a}^{s}=\sum_{w \in y} I L I_{a}^{s}(w ; y) / r_{a}$. Note that, as we have estimates of the reporting rate for only two age groups (0-14 and 15+ years) but ILI cases for four age groups $\left(0-4,5-14,15-64,65+\right.$ years), we apply $r_{0-14}$ to age groups $0-4$ and 5-14years and we apply $r_{15+}$ to age groups 15-64 and 65+ years. Details are provided in the Appendix [see Section 4 in Additional File 1].

\section{Modeling analysis}

To obtain posterior estimates of the epidemic reproduction number and age-specific susceptibility to infection for each influenza type/subtype and season, we use a Bayesian approach. First, we define an ordinary differential equation influenza transmission model following the classic SEIR scheme. Essentially, susceptible individuals (S) can acquire the infection and enter the latent compartment (L) after contact with an infectious individual (I). After a latent period, latent individuals become infectious and can transmit the infection. Finally, after an infectious period, infectious individual recovers and enter the removed ( $R$ ) compartment. The latent period is set to 1.5 days [21] and the infectious period is set to 1.2 days in such a way that the resulting generation time is 2.7 days, in agreement with the literature [22].

The population is further divided into 86 age groups (1-year age groups from 0 to 84 year and one age group for individuals aged 85 years or older) to account for a heterogeneous contact pattern by age, which is well known to be a major determinant of influenza dynamics $[23,24]$. In particular, we use the age-specific contact matrix derived for the Italian population at 1-year age resolution presented in [24]. In addition, three model parameters account for the relative susceptibility to infection of individuals between 5 and 14years of age, between 15 and 64 years of age and individuals aged 65 or older with respect to individuals aged 4 or younger. These parameters capture social, hygienic, and biological determinants (e.g., residual immunity to the circulating type/subtype) of the infection, which are not captured by the heterogeneous age-mixing pattern [7, 16, 25-27]. We set the initial condition of the system by considering the observed age-specific vaccination coverage and type/ subtype-specific vaccine efficacy [20]. The model is regulated by four free parameters: the transmission rate, and the susceptibility to infection of age groups 5-14, 15-64, $65+$ years relative to the age group 0-4year (for which the susceptibility to infection is set to the reference value of 1 ).

For each season and influenza type/subtype, we explore the likelihood of observing the estimated type/subtypespecific infection attack rate $\left(A R_{a}^{s}\right)$ given a set of model parameters by using a differential evolution Markov chain Monte Carlo (MCMC) approach [28]. To address potential bias due to paucity of data that may also affect the goodness of MCMC convergence, we report results only for the types/subtypes accounting for at least $6 \%$ of the samples testing positive for influenza. The posterior distribution of the (season- and type/subtype-specific) 
effective reproduction number $R_{\text {eff }}$ is then computed at the beginning of the season by using the next-generation matrix approach [29]. Specifically, $R_{\text {eff }}$ was computed as the dominant eigenvalue of the Next Generation Matrix (NGM) [29] associated with the dynamical system considered, accounting not only for age patterns in the Italian contact matrix but also for the susceptibility to infection among different age classes. In other words, $R_{\text {eff }}$ represents the number of secondary infections averaged over the age distribution of the typical infector for each season and influenza type/subtype and accounts for the partial immunity of the population at the onset of the influenza season. Details on the methodology are reported in the Appendix [see Sections 5 and 6 in Additional File 1].

\section{Results}

In each of the ten analyzed seasons, the incidence of ILI cases reported to the Italian surveillance system shows an annual epidemic characterized by a peak occurring in February with the exception of the 2016-2017 season when the peak was recorded in December (Fig. 1A). The maximum peak week incidence varied from 6.1 cases per 1000 individuals in the $2015-2016$ season to 14.7 cases per 1000 individuals in the 2017-2018 season (Fig. 1A). The share of ILI cases testing positive for influenza ranges from $22.9 \%$ in the $2013-2014$ season and $42.6 \%$ in the 2010-2011 season (Fig. 1B). All the analyzed seasons are characterized by the co-circulation of A/H1N1pdm09, A/ $\mathrm{H} 3 \mathrm{~N} 2$, and B types/subtypes, although in some seasons most samples tested positive for one virus only (e.g., in the 2011-2012 season, 96\% of the samples testing positive for influenza are associated with $\mathrm{A} / \mathrm{H} 3 \mathrm{~N} 2$ infection), while in other seasons all the types/subtypes showed a similar share (e.g., in the 2019-2020 season) [see Table S3 in Additional File 1].

The estimated reporting rates of the Italian surveillance system are $r_{0-14}=0.184$ (95\%CI: $\left.0.164-0.208\right)$ and $r_{15+}=0.293$ (95\%CI: 0.204-0.445) for individuals aged $0-14$ years and $15+$ years, respectively. These values are in general agreement with independent estimates available in the literature $[5,25,30]$. When accounting for the reporting rate, we have a clearer picture of the actual magnitude of the seasonal influenza epidemics by type/ subtype (Fig. 1C). The peak week incidences ranged from 5.8 influenza infections per 1000 individuals $(95 \% \mathrm{CI}$ : 5.0-7.0 infections per 1000 individuals) to 19.8 influenza infections per 1000 individuals (95\%CI: 16.9-25.0 infections per 1000 individuals). For each season, the infection attack rate (all types/subtypes) ranged from 12.7\% (95\%CI: $10.7-15.9 \%$ ) in the 2016-2017 season to $30.5 \%$ (95\%CI: 26.8-36.5\%) in the 2017-2018 season (Fig. 2A). The type/subtype-specific infection attack rate is instead highly variable across seasons (Fig. 2B-D). The largest infection attack rate for A/H3N2 influenza was $22.6 \%$ (95\%CI: 19.3-27.3\%), observed during the 2011-2012 season; for B it was $18.3 \%$ (95\%CI: $15.7-21.9 \%$ ) in the 2012-2013 season; for A/H1N1pdm09 it was $14.1 \%$ (95\%CI: $12.3-16.9 \%$ ) in the $2017-2018$ season. Interestingly, the second largest infection attack rate for $\mathrm{A} /$ H1N1pdm09 was recorded the season following the pandemic, $12.4 \%$ (95\%CI: $8.3-18.1 \%)$, and it was slightly

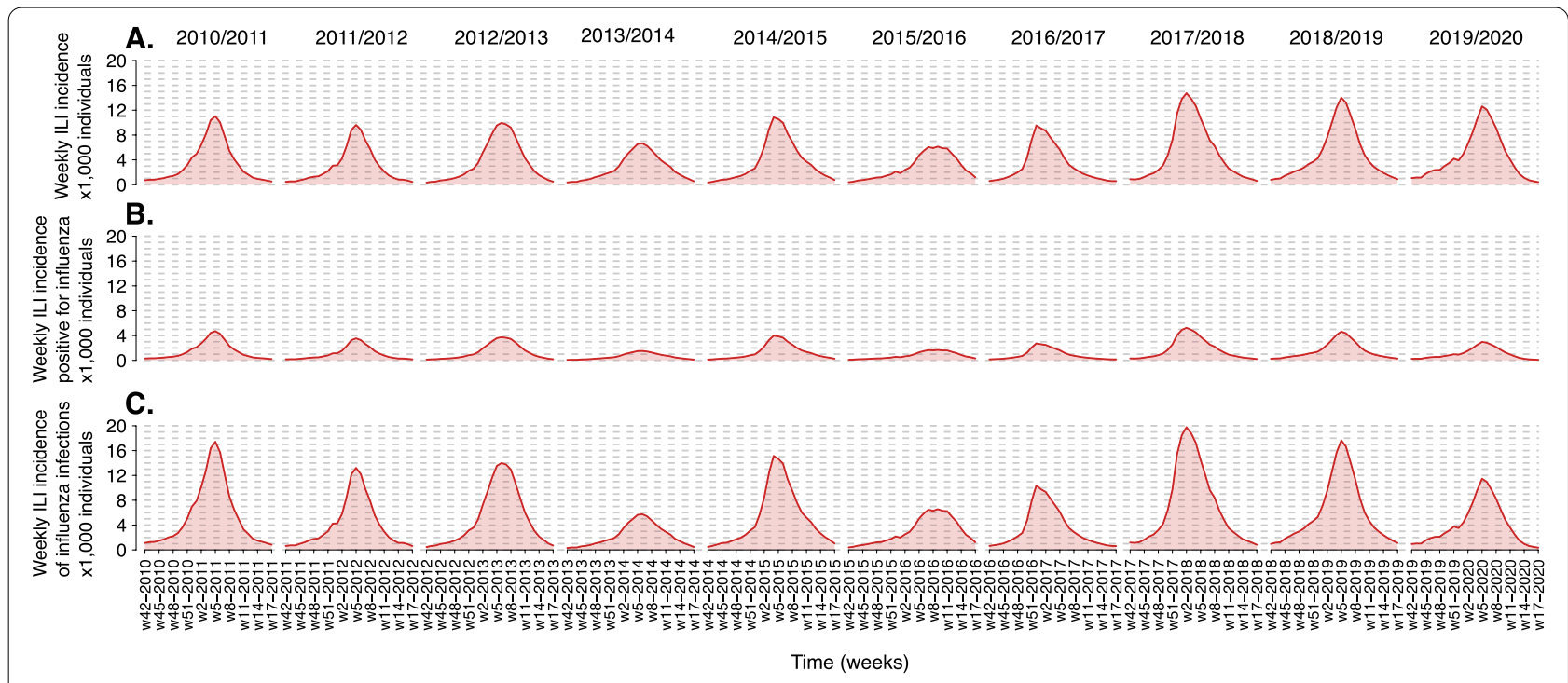

Fig. 1 A Weekly incidence of ILI cases per 1000 individuals reported to the Italian surveillance system for the ten seasons from 2010 to 2011 to 2019-2020. B As A, but for ILI cases testing positive for influenza virus. C As B, but for the estimated incidence of influenza infections, adjusted by considering the reporting rate 


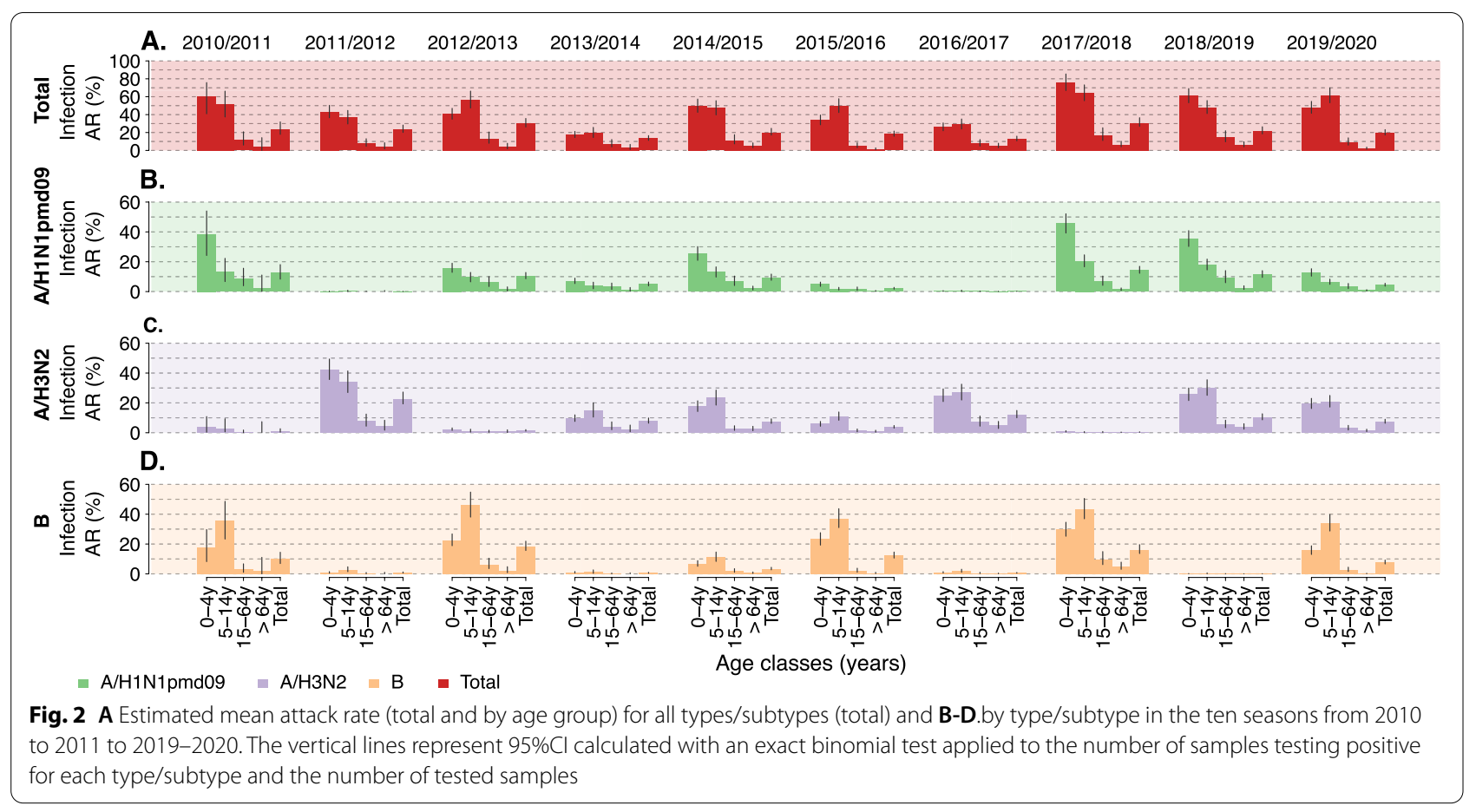

lower than that observed during the pandemic year, 16.3\% (95\%CI: 9.4-23.1\%) [16]. Essentially no circulation of A/H1N1pdm09 was recorded in 2011-2012 and 2016-2017, while low circulation was observed in the 2013-2014, 2015-2016 and 2019-2020 seasons (estimated infection attack rates lower than 5\%).

By considering infection attack rates by age, the figure is very heterogeneous across different seasons and influenza subtype (Fig. 2B-D). Specifically, for A/H1N1 subtype, the infection attack rate decreases by age group, while for A/H3N2 this hold only in the 2011-2012 season, when it is the predominant subtype. During seasons when all three types/subtypes co-circulated, i.e., 2014-2015, 2015-2016, and 2019-2020, individuals aged $5-14$ years were the most affected by A/H3N2. On the other side, in all the analyzed seasons, the most affected age group by influenza B is $5-14$ years with attack rates as high as $46.3 \%$ (95\%CI: 38.1-54.8\%) during the 2012-2013 season. The elderly appear to be the least affected segment of the population across all types/subtypes and seasons, showing an attack rate consistently lower than $5.0 \%$.

We estimate the effective reproduction number to be in the range 1.08-1.41 (see Table. 1), in agreement with the literature on seasonal influenza [31]. We find that the reproduction number of $\mathrm{A} / \mathrm{H} 1 \mathrm{~N} 1 \mathrm{pdm} 09$ was highest in 2017-2018 season and reached the value of 1.41 (95\%CI: 1.37-1.46), lower than values observed in Italy for the pandemic [5]. Only during three seasons the mean effective reproduction number exceeded 1.3: for $\mathrm{A} / \mathrm{H} 3 \mathrm{~N} 2$
Table 1 Estimated posterior distribution of the effective reproduction number (mean and $95 \% \mathrm{Cl}$ ). Only types/subtypes that accounted for more than $15 \%$ of the seropositive samples are considered

\begin{tabular}{|c|c|c|c|}
\hline \multirow[t]{3}{*}{ Season } & \multicolumn{3}{|c|}{ Mean effective reproduction number $(95 \% \mathrm{Cl})$} \\
\hline & \multicolumn{3}{|c|}{ Influenza type/subtype } \\
\hline & A/H1N1pdm09 & A/H3N2 & B \\
\hline 2010-2011 & $1.29(1.16-1.5)$ & - & $1.28(1.19-1.38)$ \\
\hline 2011-2012 & - & $1.31(1.27-1.34)$ & - \\
\hline $2012-2013$ & $1.11(1.1-1.12)$ & - & $1.33(1.29-1.38)$ \\
\hline 2013-2014 & $1.1(1.09-1.11)$ & $1.11(1.09-1.12)$ & - \\
\hline 2014-2015 & $1.14(1.12-1.15)$ & $1.14(1.12-1.16)$ & $1.1(1.08-1.11)$ \\
\hline $2015-2016$ & $1.08(1.07-1.1)$ & $1.09(1.08-1.11)$ & $1.25(1.22-1.27)$ \\
\hline 2016-2017 & - & $1.16(1.14-1.17)$ & - \\
\hline $2017-2018$ & $1.41(1.37-1.46)$ & - & $1.27(1.25-1.3)$ \\
\hline 2018-2019 & $1.22(1.2-1.24)$ & $1.19(1.17-1.21)$ & - \\
\hline 2019-2020 & $1.11(1.09-1.12)$ & $1.14(1.13-1.15)$ & $1.23(1.22-1.25)$ \\
\hline
\end{tabular}

subtype in the 2011-2012 season (estimated mean 1.31, 95\%CI: 1.27-1.34) and for B virus in the 2012-2013 season (estimated mean 1.33 95\%CI: 1.29-1.38).

We estimate the susceptibility to infection to be rather constant across different seasons, but rather heterogeneous across different types/subtypes, suggesting that this epidemiological parameter is highly characteristic of each type/subtype (Fig. 3). In all seasons, we estimate 

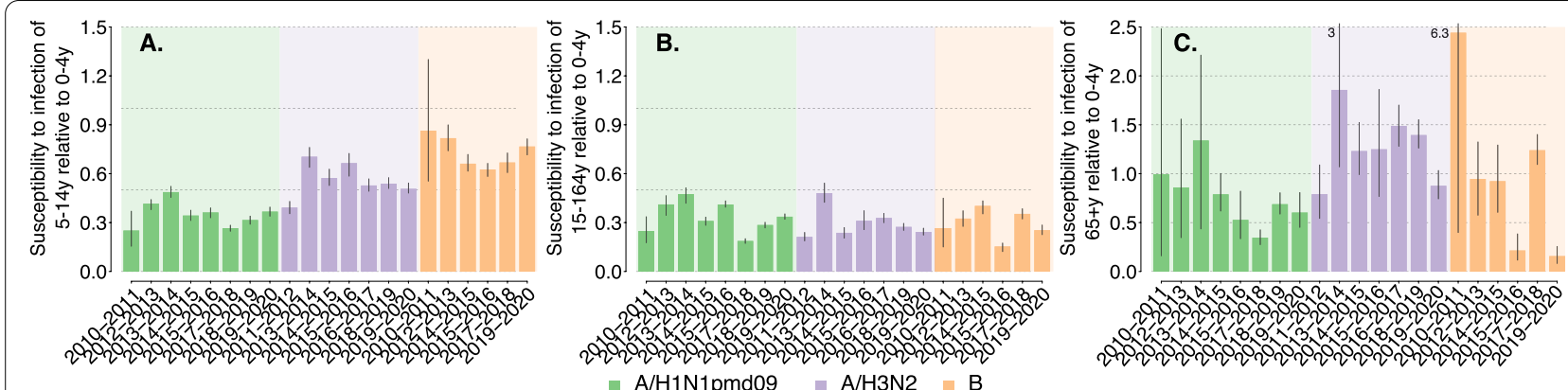

Fig. 3 Estimated posterior distributions of the susceptibility to infection of 5-14y $(\mathbf{A}), 15-64 \mathrm{y}(\mathbf{B})$ and $65+\mathrm{y}(\mathbf{C})$ age group (mean and 95\%Cl) relative to the $0-4$ years age group (for which the susceptibility to infection is set to the reference value of 1 ). Only types/subtypes that accounted for more than $15 \%$ of the positive samples are considered. The values reported above the vertical lines in the right panels represent the $97.5 \%$ percentile of the distribution, when the value exceed the limit of the vertical axis

the susceptibility to $\mathrm{A} / \mathrm{H} 1 \mathrm{~N} 1 \mathrm{pdm} 09$ infection to be in the range $0.25-0.49$ for both 5-14years and $15-64$ years age groups, markedly different from what was estimated for the 2009 pandemic where school-age individuals were highly susceptible to infection [7, 16, 25-27, 32].

A markedly larger susceptibility to infection was estimated for individuals aged 5-14years for the other two types/subtypes, reaching values of 0.86 (95\%CI: $0.55-$ 1.33) for $B$ influenza during the 2010-2011 season and 0.70 (95\%CI: 0.64-0.76) for $\mathrm{A} / \mathrm{H} 3 \mathrm{~N} 2$ influenza during the 2013-2014 season. For all types/subtypes, a highly variable susceptibility to infection is estimated for the elderly, with mean values ranging from nearly 0.16 up to 2.44. This is due to the low number of samples collected (and also testing positive) among ILI cases aged $65+$ years, which results in highly variable Bayesian estimates. However, in the seasons where influenza circulation among the elderly is detected, the elderly tends to be more susceptible to $\mathrm{A} / \mathrm{H} 3 \mathrm{~N} 2$ and $\mathrm{B}$ infection than to $\mathrm{A} /$ H1N1pdm09 infection.

Two sensitivity analyses were conducted to assess the robustness of our results with respect to the length of the generation time. Specifically, we reported results on Reff and age-specific susceptibility to infection obtained by setting the generation time in the range 1.7-3.7 days, based on a systematic review of serial intervals of respiratory infectious diseases [22].

Estimates of all four parameters are coherent with results of the baseline analysis [see Fig.S4-S5 and Table S4-S5 in Additional File 1].

\section{Discussion}

By analyzing epidemiological and surveillance data as well as seroepidemiological, socio-demographic, and contact data, we used mathematical modeling to characterize influenza transmission patterns over ten influenza seasons in Italy. We witnessed an alternation of a predominant type/subtype and the co-circulation of multiple types/subtypes. Nonetheless, we estimate the effective reproduction number to be mostly in the range 1.1-1.3 and only in one season above 1.4, in general agreement with the literature [31]. Age-specific susceptibility to infection appears to be type/subtype-related, with a markedly larger susceptibility of 5-14 years old individuals to $\mathrm{A} / \mathrm{H} 3 \mathrm{~N} 2$ and $\mathrm{B}$ influenza infection than to A/H1N1pdm09 influenza infection. Susceptibility to infection estimates for the elderly are highly variable both by type/subtype and by season. The estimated reporting rate to the Italian national surveillance system of about 1 case out of $3-5$ is in line with the estimates found in previous studies $[5,25,30]$.

The estimated total infection attack rate varied between about $13 \%$ to about $30 \%$ across seasons.

To our knowledge, there are no studies reporting estimates on seroconversion rates for seasonal influenza neither for Italy nor for other European countries to compare our results with. However, a recent study based on data collected in central and southern Vietnam between 2009 to 2015 estimated annual attack rates at $25.6 \%$ (95\% CI: $24.1-27.1 \%)$ for $\mathrm{H} 3$ strains and at $16.0 \%$ (95\% CI: 14.7-17.3\%) for H1 strains [33], and another longitudinal study conducted in Hong Kong between 2009 and 2011 on influenza A estimated seroconversion rates as high as $22 \%$, and slightly higher among the elderly for A/ H3N2 [34], which are in line with our estimates. When analyzing the infection attack rate by age, an even larger variability was estimated, mainly associated with the different infection patterns of the three types/subtypes. In fact, individuals aged 0-4years are generally the most affected group by influenza A/H1N1pdm09 subtype while $B$ influenza virus affects mainly individuals aged 5-14 years. However, A/H1N1pdm09 and B types/subtypes affect the elderly less than $\mathrm{A} / \mathrm{H} 3 \mathrm{~N} 2$, probably due to the high degree of antigenic drift of the $\mathrm{A} / \mathrm{H} 3 \mathrm{~N} 2$ virus 
[35], and the consequent lower vaccine effectiveness against A/H3N2 infection [20] and higher susceptibility to infection. Overall, individuals aged 14 years or less are the most affected segment of the population, coherently with seroepidemiological studies performed on the 2009 pandemic [16, 36-38], probably due to both a larger susceptibility to infection, as measured in this study and in the literature $[7,16,26,27]$, and to the larger number of social contacts of school-age individuals with respect to the rest of the population [23-25].

We estimate the elderly to be the least affected age group. However, this segment of the population is associated in the literature with larger hospitalization and mortality rates $[39,40]$. Therefore, also in the light of the rapid increase in the aging of populations throughout the world, this makes the study of immunosenescence in older individuals and the development of more immunogenic vaccines, two pipeline priorities in the control and prevention of influenza [41]. Moreover, although our estimates of the age-specific susceptibility to infection cannot be used to discern between biological, social, or hygienic determinants, they provide relevant indications for interventions targeting specific age segments of the population, school-age individuals above all.

These results support the importance of recommending the vaccine also to school-age healthy children, as already done by some EU countries and internationally [42] and call for further studies on school closure strategies as a possible non-pharmaceutical intervention to mitigate influenza spread in case of severe and/or pandemic seasons [25, 43-48].

It is important to stress that our study suffer of some limitations. First, neither we explicitly consider the potential cross protection among different types/subtypes nor the co-circulation of multiple influenza types/ subtypes. However, by considering age- and season-specific susceptibility to infection to each influenza type/ subtype, the estimated infection attack rates by age are not strongly affected by the lack of an explicit simulation of cross protection and co-circulation of multiple influenza types/subtypes. To pin down the effect of cross protection alone, the collection of serological data for different influenza type/subtype of the Italian population in different age classes would be warranted. A second limitation is that we relied on reporting rates estimated during the H1N1 pandemic in Italy, which are available for two age strata of the population that encompass include individuals possibly characterized by very different risks and behaviors. However, these estimates are in general agreement with independent estimates available in the literature $[5,25,32]$. A third limitation is that we assumed that the estimated reporting rates are constant across all the analyzed seasons and types/subtypes. The calculation of type/subtype- and season-specific reporting rates was not possible due to the unavailability of the necessary data (pre- and post-influenza season serological data). Although our assumption is partially backed up by the findings of Carrat and colleagues [4], who estimated a similar share of individuals developing clinical symptoms after influenza infection for $\mathrm{A} / \mathrm{H} 1 \mathrm{~N} 1$, A/ $\mathrm{H} 3 \mathrm{~N} 2$, and B influenza types/subtypes, there is evidence of a vaccine-associated reduction of symptoms among vaccinated patients [49]. We acknowledge that the adoption of reporting rates estimated for the 2009 H1N1 pandemic, which do not account for vaccination, may affect our estimates of transmissibility and age-specific susceptibility to infection. A final limitation is that the attack rates were estimated using virological data by age over the entire course of the influenza season, without accounting for possible heterogeneities over time, due to data unavailability. Although this may bias our estimates of the effective reproduction number and susceptibility to infection for the three types/sub-types, we would like to stress that the virological surveillance is designed in such a way that the number of analyzed samples is proportional to the incidence of reported ILIs. Indeed, the general aim of virological surveillance is to facilitate vaccine strain selection by understanding the circulating influenza virus types, subtypes, and lineages and the age distribution of influenza confirmed cases [50].

\section{Conclusions}

Our study provides indications on age- and type/ subtype-specific incidence rates and susceptibility to infection as well as pathogen transmissibility, thus contributing to define a clear picture of the epidemiology of seasonal influenza in Italy. Our work provides relevant insights on age-specific targets for influenza immunization and non-pharmaceutical intervention plans, possibly also tailored on the circulating influenza type/subtype.

\section{Abbreviations}

ILI: Influenza-like illness; GP: General practitioner; PD: Pediatrician; CRF: Case report form; NIC: National influenza center; ISS: Italian National Institute of Health; AR: Attack rate; MCMC: Markov Chain Monte Carlo; NGM: Next Generation Matrix; $R_{\text {eff: }}$ Effective reproduction number.

\section{Supplementary Information}

The online version contains supplementary material available at https://doi. org/10.1186/s12889-021-12426-9.

Additional file 1. Additional results.

\section{Acknowledgments}

Not applicable. 


\section{Role of the funder/sponsor}

Seqirus had a possibility to review the study protocol but had no role in any other aspect of the study conduct. In particular, Seqirus had no role in the design and conduct of the study, the collection, management, analysis, and interpretation of the data, or the preparation, or approval of the manuscript, and decision to submit for publication.

\section{Authors' contributions}

Study design: FT, CR, SM, MA. Acquisition of data: EP, AB, GD, LC, CR. Data analysis: FT. Interpretation of results: FT, CR, SM, MA. Drafting of the manuscript: FT, MA. Critical revision of the manuscript: EP, AB, CR, SM. All authors have read and approved the final manuscript.

\section{Funding}

F.T. and S.M. acknowledge funding from the European Commission H2O2O project MOOD and from the VRT Foundation Trento project "Epidemiologia e transmissione di COVID-19 in Trentino". The present study received an unconditional funding from Segirus.

\section{Availability of data and materials}

The datasets used and/or analysed during the current study available from the corresponding.

author on reasonable request.

\section{Declarations}

\section{Ethics approval and consent to participate}

All data analyzed in this study is collected by the Italian Institute of Health within the surveillance of seasonal influenza and is publicly available. All methods were carried out in accordance with relevant guidelines and regulations.

\section{Consent for publication}

Not applicable.

\section{Competing interests}

M.A. has received consultancy fees from Seqirus. All other authors declare no competing interests.

\section{Author details}

${ }^{1}$ Center for Health Emergencies, Bruno Kessler Foundation, Trento, Italy. ${ }^{2}$ Dondena Centre for Research on Social Dynamics and Public Policy, Bocconi University, Milan, Italy. ${ }^{3}$ Dipartimento di Scienze Biomediche per la Salute, Università degli Studi di Milano, Milan, Italy. ${ }^{4}$ Department of Infectious Diseases, Italian National Institute of Health (ISS), Rome, Italy. ${ }^{5}$ General Directorate for Health Planning, Ministry of Health, Rome, Italy. ${ }^{6}$ Unità Organizzativa Prevenzione, Regione Lombardia, Milan, Italy. ${ }^{7}$ Clinical Pathways and Epidemiology Functional Area, Bambino Gesù Children's Hospital, IRCCS IT, Rome, Italy. ${ }^{8}$ Laboratory for Computational Epidemiology and Public Health, Department of Epidemiology and Biostatistics, Indiana University School of Public Health, Bloomington, IN, USA.

Received: 29 November 2020 Accepted: 14 December 2021 Published online: 06 January 2022

\section{References}

1. Gordon A, Reingold A. The burden of influenza: a complex problem. Curr Epidemiol Rep. 2018;5(1):1-9.

2. Iuliano AD, Roguski KM, Chang HH, et al. Estimates of global seasonal influenza-associated respiratory mortality: a modelling study. Lancet. 2018;391(10127):1285-300.

3. Carrat F, Flahault A. Influenza vaccine: the challenge of antigenic drift. Vaccine. 2007;25(39-40):6852-62.

4. Carrat F, Vergu E, Ferguson NM. at al. Time lines of infection and disease in human influenza: a review of volunteer challenge studies. Am Epidemiol. 2008;167(7):775-85.

5. Ajelli M, Merler S, Pugliese A, et al. Model predictions and evaluation of possible control strategies for the 2009 A/H1 N1v influenza pandemic in Italy. Epidemiol Infect. 2011;139(1):68-79.
6. Cauchemez S, Carrat F, Viboud C, et al. A Bayesian MCMC approach to study transmission of influenza: application to household longitudinal data. Stat Med. 2004:23(22):3469-87.

7. Cauchemez S, Donnelly CA, Reed C, et al. Household transmission of 2009 pandemic influenza A (H1N1) virus in the United States. N Engl J Med. 2009;361(27):2619-27.

8. Birrell PJ, Ketsetzis G, Gay NJ, et al. Bayesian modeling to unmask and predict influenza A/H1N1pdm dynamics in London. Proc Natl Acad Sci. 2011;108(45):18238-43.

9. Marziano V, Pugliese A, Merler S, et al. Detecting a Surprisingly Low Transmission Distance in the Early Phase of the 2009 Influenza Pandemic. Sci Rep. 2017;7(1):12324.

10. Istituto Superiore di Sanità. Sistema di Sorveglianza Sentinella dell'Influenza basata su Medici di Medicina Generale, Pediatri di Libera Scelta e Laboratori di Riferimento Regionale per l'Influenza. Protocollo Operativo. Available at http://www.salute.gov.it/imgs/C_17_pubblicazi oni_2889_allegato.pdf. Accessed on 24 Oct 2021.

11. Istituto Superiore di Sanità. Operative protocol for virological surveillance. Available at: https://w3.iss.it/site/RMI/influnet/pagine/Docum enti.aspx. Accessed on 24 Oct 2021.

12. Italian Ministry of Health. Prevenzione e controllo dell'influenza. Available at: https://www.trovanorme.salute.gov.it/norme/renderNormsanPdf?anno $=2020 \&$ codLeg $=74451 \&$ parte $=1 \% 20 \&$ serie $=$ null. Accessed on 24 Oct 2021.

13. Pariani E, Amendola A, Piatti A, et al. Ten years (2004-2014) of influenza surveillance in Northern Italy. Hum Vacc Immunother. 2015:11(1):198-205.

14. Puzelli S, Di Martino A, Facchini M, et al. Co-circulation of the two influenza B lineages during 13 consecutive influenza surveillance seasons in Italy, 2004-2017. BMC Infect Dis. 2019;19(1):990.

15. Rizzo C, Rota MC, Bella A, et al. Cross-reactive antibody responses to the 2009 A/H1N1v influenza virus in the Italian population in the prepandemic period. Vaccine. 2010;28(20):3558-62.

16. Merler S, Ajelli M, Camilloni B, et al. Pandemic influenza A/ H1N1pdm in Italy: age, risk and population susceptibility. Plos One. 2013;8(10):e74785.

17. Istituto Superiore di Sanità. Epidemiological data on influenza. Available at: https://www.epicentro.iss.it/influenza/dati. Accessed on 24 Oct 2021.

18. ISTAT. Health for All - Italy. Available at: https://www.istat.it/it/archivio/ 14562. Accessed on 24 Oct 2021.

19. ISTAT. Statistiche demografiche. Available at: http://demo.istat.it/index e. html. Accessed on 24 Oct 2021.

20. Belongia EA, Simpson MD, King JP, et al. Variable influenza vaccine effectiveness by subtype: a systematic review and meta-analysis of testnegative design studies. Lancet Infect Dis. 2016;16(8):942-51.

21. Lessler J, Reich NG, Brookmeyer R, et al. Incubation periods of acute respiratory viral infections: a systematic review. Lancet Infect Dis. 2009:9(5):291-300

22. Vink MA, Bootsma MCJ, Wallinga J. Serial intervals of respiratory infectious diseases: a systematic review and analysis. Am J Epidemiol. 2014;180(9):865-75

23. Mossong J, Hens $N$, Jit M, et al. Social contacts and mixing patterns relevant to the spread of infectious diseases. Plos Med. 2008;5(3):e74.

24. Fumanelli $L$, Ajelli $M$, Manfredi $P$, et al. Inferring the structure of social contacts from demographic data in the analysis of infectious diseases spread. Plos Comput Biol. 2012;8(9):e1002673.

25. Litvinova M, Liu Q-H, Kulikov ES, et al. Reactive school closure weakens the network of social interactions and reduces the spread of influenza. Proc Natl Acad Sci USA. 2019;116(27):13174-13,181.

26. Fraser C, Donnelly CA, Cauchemez S, et al. Pandemic potential of a strain of influenza A (H1N1): early findings. Science. 2009;324(5934):1557-61.

27. Ajelli M, Poletti P, Melegaro A, et al. The role of different social contexts in shaping influenza transmission during the 2009 pandemic. Sci Rep. 2014; $4: 7218$.

28. Turner BM, Sederberg PB, Brown SD, et al. A method for efficiently sampling from distributions with correlated dimensions. Psychol Methods. 2013;18(3):368

29. Diekmann O, Heesterbeek JA, Metz JA. On the definition and the computation of the basic reproduction ratio $\mathrm{RO}$ in models for infectious diseases in heterogeneous populations. J Math Biol. 1990;28(4):365-82. 
30. McDonald SA, van Boven M, Wallinga J. An evidence synthesis approach to estimating the proportion of influenza among influenza-like illness patients. Epidemiol. 2017;28(4):484-91.

31. Merler S, Ajelli M, Pugliese A, et al. Determinants of the spatiotemporal dynamics of the $2009 \mathrm{H} 1 \mathrm{~N} 1$ pandemic in Europe: implications for realtime modelling. Plos Comput Biol. 2011;7(9):e1002205.

32. Biggerstaff $M$, Cauchemez $S$, Reed $C$, et al. Estimates of the reproduction number for seasonal, pandemic, and zoonotic influenza: a systematic review of the literature. BMC Infect Dis. 2014;14(1):480.

33. Vinh DN, Nhat NTD, de Bruin E, Vy NHT, Thao TTN, et al. Age-seroprevalence curves for the multi-strain structure of influenza A virus. Nat Commun. 2021;12(1):6680

34. Kwok KO, Riley S, Perera RAPM, Wei VWI, Wu P, et al. Relative incidence and individual-level severity of seasonal influenza A H3N2 compared with 2009 pandemic H1N1. BMC Infect Dis. 2017;17(1):337.

35. Tewawong N, Prachayangprecha S, Vichiwattana P, et al. Assessing antigenic drift of seasonal influenza A (H3N2) and A (H1N1) pdm09 viruses. Plos One. 2015;10(10):e0139958.

36. Hardelid P, Andrews NJ, Hoschler K, et al. Assessment of baseline age-specific antibody prevalence and inci- dence of infection to novel influenza A/H1N1 2009. Health Technol Assess. 2010;14(55):115-92.

37. Reed C, Katz JM, Hancock K, et al. Prevalence of seropositivity to pandemic influenza A/H1N1 virus in the United States following the 2009 pandemic. Plos One. 2012;7(10):e48187.

38. Weil $M$, Shohat $T$, Bromberg $M$, et al. The dynamics of infection and the persistence of immunity to A (H1N1) pdm09 virus in Israel. Influenza Other Respir Viruses. 2013;7(5):838-46.

39. Simonsen L, Taylor RJ, Viboud C, et al. Mortality benefits of influenza vaccination in elderly people: an ongoing controversy. Lancet Infect Dis. 2007;7(10):658-66.

40. Rizzo C, Bella A, Viboud C, et al. Trends for influenza-related deaths during pandemic and epidemic seasons, Italy, 1969-2001. Emerg Infect Dis. 2007;13(5):694.

41. Lambert ND, Ovsyannikova IG, Pankratz VS, et al. Understanding the immune response to seasonal influenza vaccination in older adults: a systems biology approach. Exp Rev Vacc. 2012;11 (8):985-94.

42. Rizzo C, Rezza G, Ricciardi W. Strategies in recommending influenza vaccination in Europe and US. Hum Vacc Immunother. 2018;14(3):693-8.

43. Cowling BJ, Lau EH, Lam CL, et al. Effects of school closures, 2008 winter influenza season, Hong Kong. Emerg Infect Dis. 2008;14(10):1660.

44. Wu JT, Cowling BJ, Lau EH, et al. School closure and mitigation of pandemic (H1N1) 2009, Hong Kong. Emerg Infect Dis. 2010;16(3):538.

45. House T, Baguelin M, Van Hoek AJ, et al. Modelling the impact of local reactive school closures on critical care provision during an influenza pandemic. Proc R Soc B. 2011;278(1719):2753-60.

46. Fumanelli L, Ajelli M, Merler S, et al. Model-based comprehensive analysis of school closure policies for mitigating influenza epidemics and pandemics. Plos Comput Biol. 2016;12(1):e1004681.

47. Ciavarella C, Fumanelli L, Merler S, et al. School closure policies at municipality level for mitigating influenza spread: a model-based evaluation. BMC Infect Dis. 2016;16(1):576.

48. De Luca G, Van Kerckhove K, Coletti P, et al. The impact of regular school closure on seasonal influenza epidemics: a data-driven spatial transmission model for Belgium. BMC Infect Dis. 2018;18(1):29.

49. Deiss RG, Arnold JC, Chen WJ, Echols S, Fairchok MP, Schofield C, et al. Vaccine-associated reduction in symptom severity among patients with influenza A/H3N2 disease. Vaccine. 2015;33(51):7160-7.

50. WHO. WHO Interim Global - Epidemiological Surveillance Standards for Influenza. Available at: https://www.who.int/influenza/resources/docum ents/INFSURVMANUAL.pdf Accessed on 24 Oct 2021.

\section{Publisher's Note}

Springer Nature remains neutral with regard to jurisdictional claims in published maps and institutional affiliations.

Ready to submit your research? Choose BMC and benefit from:

- fast, convenient online submission

- thorough peer review by experienced researchers in your field

- rapid publication on acceptance

- support for research data, including large and complex data types

- gold Open Access which fosters wider collaboration and increased citations

- maximum visibility for your research: over $100 \mathrm{M}$ website views per year

At BMC, research is always in progress.

Learn more biomedcentral.com/submissions 